\title{
Apatinib versus sorafenib in patients with advanced hepatocellular carcinoma: a preliminary study
}

\author{
Wei He ${ }^{1,2 \#}$, Leen Liao ${ }^{1,3 \#}$, Dandan $\mathrm{Hu}^{1,2}$, Binkui $\mathrm{Li}^{1,2}$, Chenwei Wang ${ }^{1,2}$, Jiliang Qiu ${ }^{1,2}$, Yadi Liao ${ }^{1,2}$, \\ Wenwu Liu ${ }^{1,2}$, Zhiwen Yang ${ }^{1,2}$, Yuanping Zhang ${ }^{1,2}$, Yichuan Yuan ${ }^{1,2}$, Kai Li $^{1,2}$, Yunfei Yuan ${ }^{1,2}$, Yun Zheng ${ }^{1,2}$ \\ ${ }^{1}$ State Key Laboratory of Oncology in South China, ${ }^{2}$ Department of Hepatobiliary Oncology, Sun Yat-sen University Cancer Center, ${ }^{3}$ Department \\ of Colorectal Surgery, Sun Yat-sen University Cancer Center, Guangzhou, China \\ Contributions: (I) Conception and design: Y Zheng, W He; (II) Administrative support: Y Zheng, Y Yuan; (III) Provision of study materials or patients: \\ Y Zheng ; (IV) Collection and assembly of data: L Liao, D Hu, C Wang, J Qiu, Y Liao, W Liu, Z Yang, Y Zhang, Y Yuan, K Li; (V) Data analysis \\ and interpretation: L Liao, W He, D Hu, W Liu, Z Yang; (VI) Manuscript writing: W He, L Liao, Y Zheng; (VII) Final approval of manuscript: All \\ authors. \\ \#These authors contributed equally to this work. \\ Correspondence to: Yun Zheng. Department of Hepatobiliary Oncology, Sun Yat-sen University Cancer Center, 651 Dongfeng Road E. Guangzhou, \\ China. Email: zhengyun@sysucc.org.cn.
}

Background: Apatinib, a selective inhibitor of vascular endothelial growth factor receptor 2 (VEGFR 2), has exhibited modest antitumor efficacy in hepatocellular carcinoma (HCC). We aimed to evaluate the effectiveness and tolerability of apatinib versus sorafenib in patients with advanced HCC.

Methods: All patients with advanced HCC who underwent sorafenib or apatinib between January 2016 to December 2017 were retrospectively reviewed. Seventy-two patients received apatinib (26 patients, $500 \mathrm{mg}$, daily) or sorafenib (46 patients, $400 \mathrm{mg}$, twice daily) until disease progression or intolerable toxicities. Primary outcome was progression-free survival (PFS). Secondary outcomes included overall survival (OS), objective response rate (ORR) per modified response evaluation criteria in solid tumors (RECIST), disease control rate (DCR), and safety.

Results: The median follow-up was 13.2 (5.7-20.7) months. The 1-year OS for apatinib of $62.0 \%$ was comparable to that of sorafenib [64.2\%, hazard ratio (HR), 1.15; 95\% confidence interval (CI), 0.369-3.58]. The median PFS was 4.1 months in the apatinib group (95\% CI, 3.2 to 7.4 months) and 3.6 months in the sorafenib group (95\% CI, 2.7 to 5.9 months; HR, 1.03; 95\% CI, 0.586 to $1.800 ; \mathrm{P}=0.925$ ). The apatinib group exhibited higher ORR (19.2\% vs. $2.2 \%, \mathrm{P}=0.012)$ but similar DCR $(57.7 \%$ vs. $50 \%, \mathrm{P}=0.530)$ compared with the sorafenib group. The most common any-grade adverse events in the apatinib and sorafenib groups were hand and foot syndrome (53.8\% vs. 50\%), hypertension $(50 \%$ vs. $19.6 \%)$, diarrhea (34.6\% vs. $28.3 \%$ ), and elevated transaminase ( $57.7 \%$ vs. $63 \%)$.

Conclusions: Compared with sorafenib, apatinib yielded comparable PFS and OS, and even better ORR, in patients with advanced HCC.

Keywords: Hepatocellular carcinoma (HCC); systemic treatment; apatinib; sorafenib

Submitted Jun 16, 2020. Accepted for publication Aug 10, 2020.

doi: $10.21037 /$ atm-20-5298

View this article at: http://dx.doi.org/10.21037/atm-20-5298

\section{Introduction}

Liver cancer is the fourth leading cause of cancer-related mortality worldwide, and hepatocellular carcinoma (HCC) accounts for approximately $90 \%$ of all primary hepatic malignancies $(1,2)$. Despite recent advances in drug development, an estimated $70-85 \%$ of HCC patients diagnosed with advanced stage 2 have poor prognosis $(3,4)$. Meanwhile, it has been reported that vascular endothelial 
growth factor (VEGF) plays an important role in the development and progression of HCC, and (5) thus, molecularly targeted therapy, especially agents targeting the VEGF and VEGF receptor, has occupied a pivotal role in the treatment of advanced HCC.

Sorafenib, a multitargeted small molecule tyrosine-kinase inhibitor (TKI) of the Raf kinase and the VEGF receptor (VEGFR), has been widely used as the first-line therapy for advanced HCC and recommended in Barcelona-Clinic Liver Cancer, National Comprehensive Cancer Network and Chinese Society of Clinical Oncology guidelines. However, the objective response rate (ORR) (2-3.3\%) evaluated by the response evaluation criteria in solid tumor (RECIST) has been found to be low, with survival benefit being modest (2.8 and 2.3 months) in both Western and Asian populations $(6,7)$. For more than ten years, sorafenib, was the only systemic agent available with a survival benefit for advanced HCC. Four failed phase 3 trials (sunitinib, brivanib, linifanib, and erlotinib plus sorafenib) did not show non-inferiority or superiority to sorafenib in terms of overall survival (OS) in first-line treatment of HCC (8-11). Recent years, several new drugs have showed prognosis benefit in advanced HCC, including TKI lenvatinib in the first-line and regorafenib, cabozantinib, and ramucirumab in the second-line therapy (12-15).

Apatinib (Hengrui Pharmaceutical Co., Ltd., Shanghai, China) is a new oral small anti-angiogenesis molecule that specifically binds VEGFR-2 with a 10 -fold greater binding affinity than sorafenib (16-18). In China, apatinib has been approved for the treatment of advanced gastric cancer and gastroesophageal junction adenocarcinoma patients (19). Two small pilot studies reported that apatinib could be well tolerated and effective for the treatment of advanced HCC $(20,21)$. Recently, a study showed that HCC patients who received apatinib in dose of $750 \mathrm{mg}$ once daily had promising outcome when compared with patients who received sorafenib. In our center, the initial recommended dose of apatinib was $500 \mathrm{mg}$. Having observed the positive tumor response with treatment of apatinib in our center since 2016, we aimed to compare the tumor response of apatinib versus sorafenib in patients with advanced HCC according to the modified RECIST (mRECIST) with respect to $(22,23)$ progression-free survival (PFS) and OS.

We present the following article in accordance with the STROBE reporting checklist (available at http://dx.doi. org/10.21037/atm-20-5298).

\section{Methods}

\section{Patient characteristics}

This retrospective cohort analysis included consecutive patients with advanced HCC treated with sorafenib and apatinib in our hospital between January 2016 and December 2017. The eligibility criteria were as follows: advanced HCC confirmed by pathological examination or clinical evidence according to the American Association for the Study of Liver Diseases (AASLD), Barcelona Clinic Liver Cancer stage category B, or category C HCC unamenable to locoregional resection or ablation, ChildPugh class A, an Eastern Cooperative Oncology Group performance status score (ECOG PS) 0-2, and at least one measurable lesion by computed tomography (CT) scan or magnetic resonance imaging (MRI) within 4 weeks before molecularly targeted therapy according to mRECIST. Tumor lesions located in an area subjected to previous local therapy had to show radiographic evidence of progressive disease (PD) to be considered a measurable lesion.

The exclusion criteria were the following: (I) lack of regular tumor response evaluation during the followup period; (II) transcatheter arterial chemoembolization (TACE), ablation or hepatic artery infusion chemotherapy within 4 weeks of sorafenib or apatinib treatment; (III) severe physical condition (e.g., severe cardiovascular disease, renal insufficiency).

The protocol of this retrospective designed study conformed to the ethical guidelines of the Declaration of Helsinki (as revised in 2013) and was approved by the Ethics Committee of the Sun Yat-sen University Cancer Centre (no. YB2018-08) with waiver of informed consent.

\section{Treatment and clinical follow-up}

Multi-disciplinary discussion was used to determine if patients should receive apatinib or sorafenib. Apatinib $500 \mathrm{mg}$ was orally self-administered once daily (QD), and the dose was reduced to $250 \mathrm{mg}$ QD in the case of any grade 3 or 4 adverse events (AEs). Sorafenib $400 \mathrm{mg}$ was administered orally twice daily, and the dose was reduced to $400 \mathrm{mg}$ QD or $200 \mathrm{mg}$ QD in the case of grade 3 or 4 AEs. Treatment continued until PD as defined by mRECIST, clinical progression, death, unacceptable drug-related toxicities, or refusal of treatment. Treatment interruptions and dose reduction were made based on toxicity. Patients were followed up for tumor response every 6 weeks in 


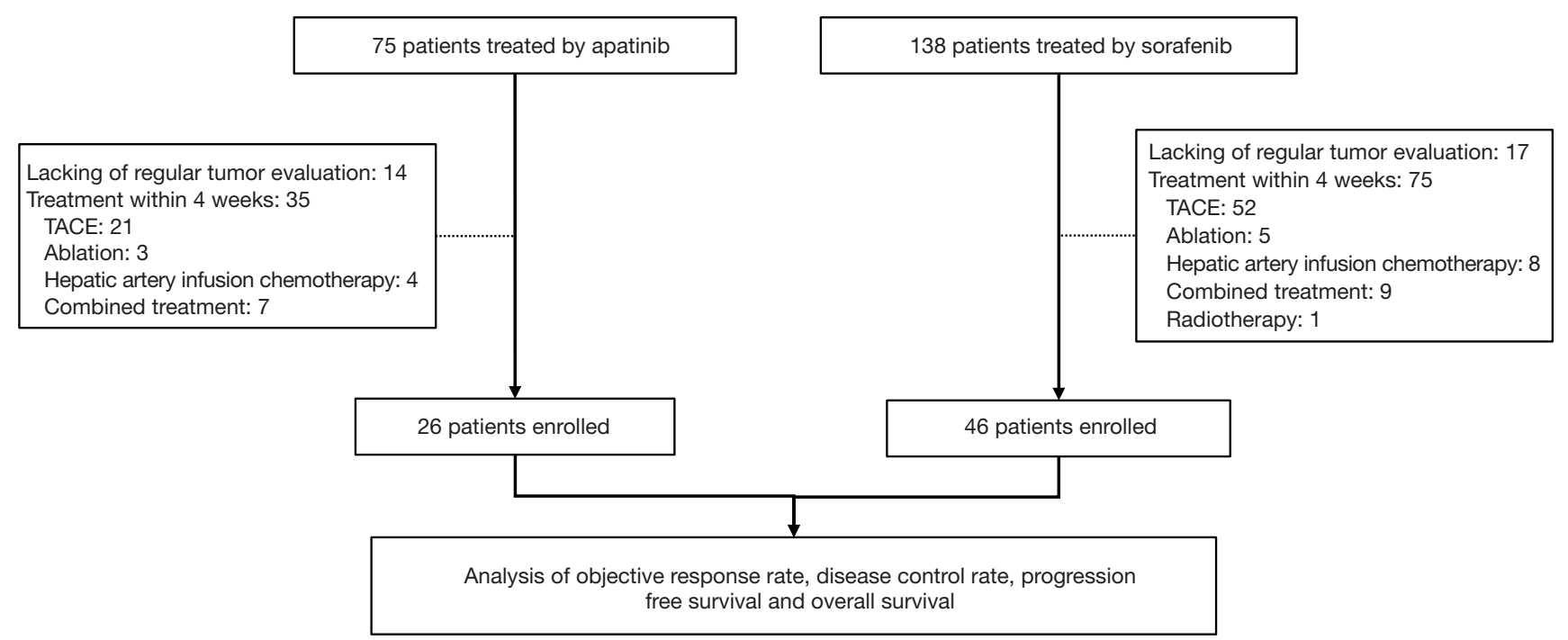

Figure 1 Flow chart.

the first 3 months and every 8 weeks thereafter. AEs were assessed according to the National Cancer Institute Common Terminology Criteria for Adverse Events (version 4.0).

PFS was defined as the time from the date of treatment to date of PD (radiological or clinical) or death due to any cause. OS was defined as the time from the date of treatment to death due to any cause. Patients still alive at the time of analysis were censored at their last date of follow-up. ORR was defined as the proportion of patients with complete response (CR) or partial response (PR) according to the mRECIST criteria. Disease control rate (DCR) was defined as the percentage of patients with CR, $\mathrm{PR}$, or stable disease (SD).

\section{Statistical analysis}

The primary outcome was PFS, while secondary outcomes included OS, ORR, DCR, and safety. Continuous variables were presented as median and interquartile range (IQR). and were compared with the independent samples $t$-test or the Mann-Whitney U-test. Qualitative variables were presented as percentage and were compared using Chi-square test or Kruskal-Wallis test. Survival curves were constructed using the Kaplan-Meier method and compared with the log-rank test. Cox proportional hazards model were used to calculate the hazard ratio (HR) with $95 \%$ confidence intervals (CI). Statistical analyses were performed using R 3.3 (www.r-project.org). P values lower than 0.05 (two-tailed) were considered significant. The key raw data in our study have been uploaded onto the Research Data Deposit public platform (www.researchdata.org.cn) (approval no. RDDA2018000566).

\section{Results}

\section{Baseline}

Between January 2016 and December 2017, 75 patients who received apatinib and 138 patients who received sorafenib treatment were identified, of whom 26 and 46 patients were finally enrolled, respectively. The remaining patients were excluded due to lacking regular tumor evaluation on CT or MRI (14 and 17 cases, respectively), or for having TACE, ablation, hepatic artery infusion chemotherapy, radiotherapy, or combined therapy within 4 weeks of apatinib and sorafenib treatment (35 and 75 cases) (Figure 1). There were no significant differences between the two treatment groups with respect to demographics characteristics, tumor burden, and liver functions (Table 1). Furthermore, 15 (57.7\%) patients in the apatinib group and $25(54.3 \%)$ patients in the sorafenib group had extrahepatic metastasis, while $12(46.2 \%)$ patients in the apatinib group and $20(43.5 \%)$ patients in the sorafenib group had major vascular invasion

\section{Treatment efficacy}

No patient in either treatment group had a CR according 
Table 1 Demographic and disease characteristics at baseline of patients with advanced hepatocellular carcinoma

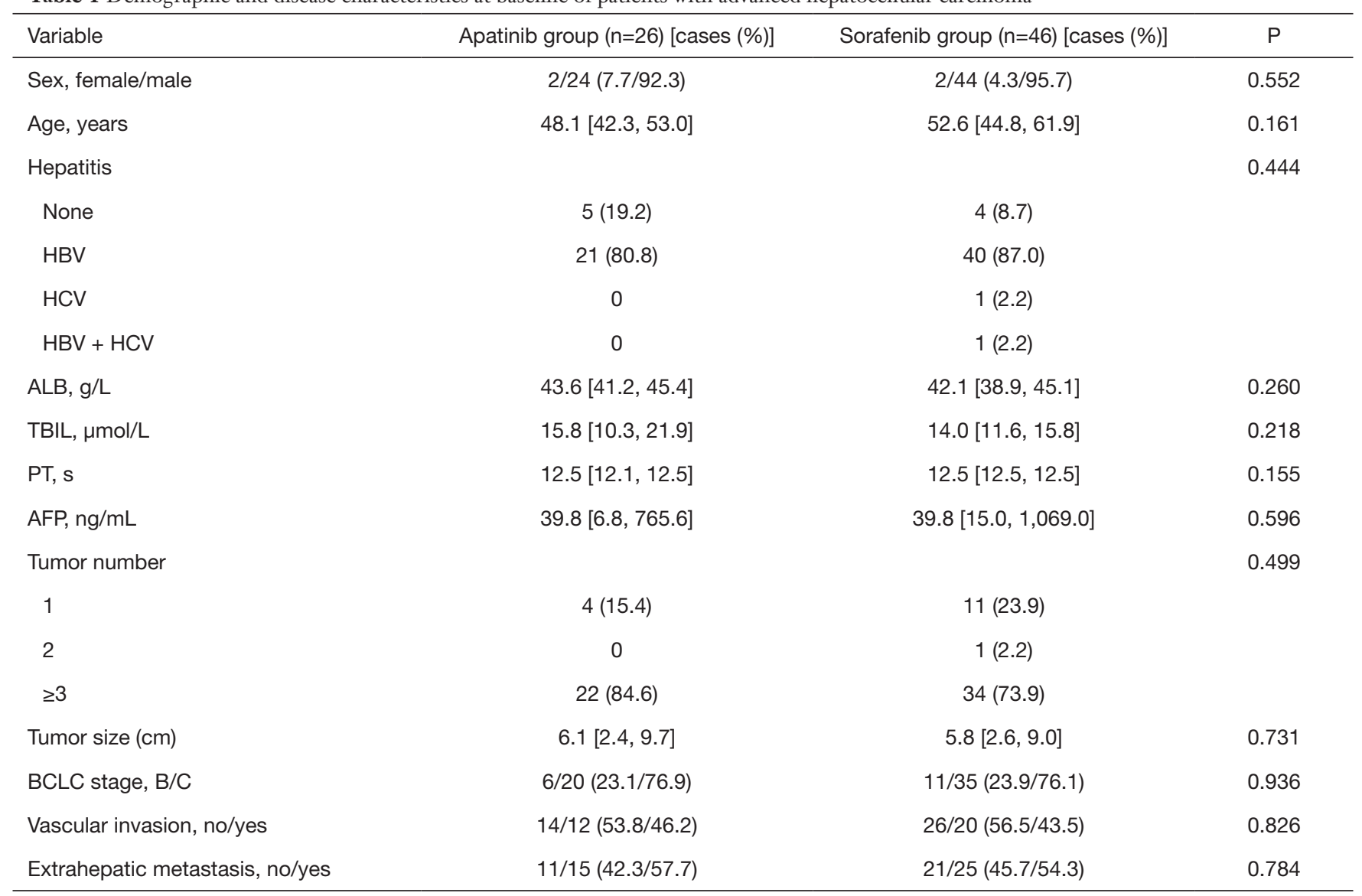

Values are presented as the median [IQR] or $\mathrm{n}(\%)$. AFP, alpha-fetoprotein; ALB, albumin; BCLC stage, Barcelona clinic liver cancer stage; HBV, hepatitis B virus; HCV, hepatitis C virus; TBIL, total bilirubin; PT, prothrombin time.

Table 2 Best tumor responses for patients with advanced hepatocellular carcinoma who underwent either apatinib or sorafenib

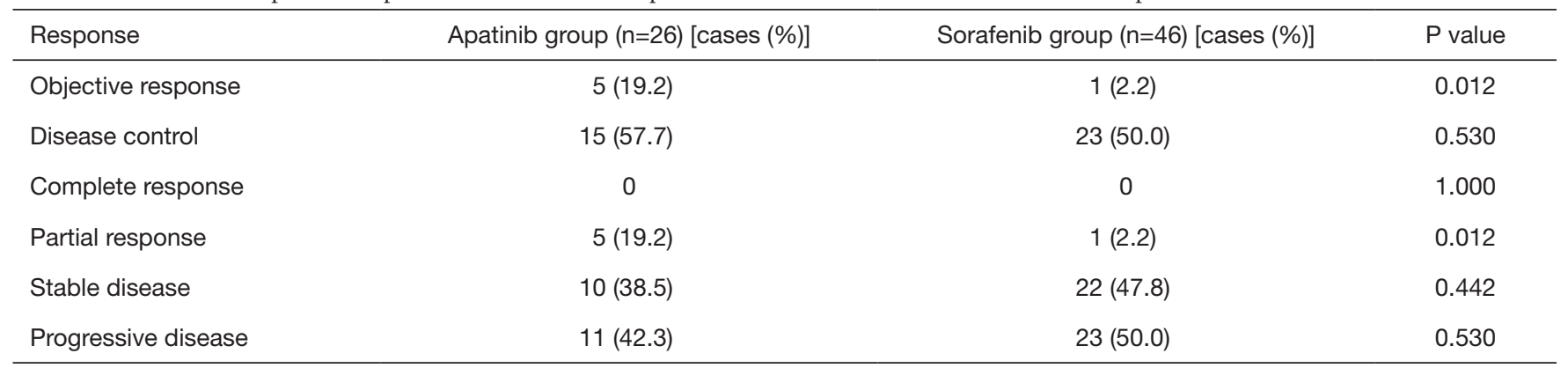

$P$ values were calculated using a two-sided Chi-square test

to mRECIST (Table 2). In total, 5 (19.2\%) patients in the apatinib group versus $1(2.2 \%)$ patient in the sorafenib group had a $\mathrm{PR}(\mathrm{P}=0.012) ; 10(38.5 \%)$ patients in the apatinib group versus $22(47.8 \%)$ patients in the sorafenib group had a $\mathrm{SD}(\mathrm{P}=0.442) ; 11(42.3 \%)$ patients in the apatinib group versus $23(50 \%)$ patients in the sorafenib group had a $\mathrm{PD}(\mathrm{P}=0.530)$; and $15(57.7 \%)$ patients in the apatinib group and $23(50 \%)$ patients in the sorafenib group achieved disease control $(\mathrm{P}=0.530)$. (Representative images in Figures 2 and 3). 

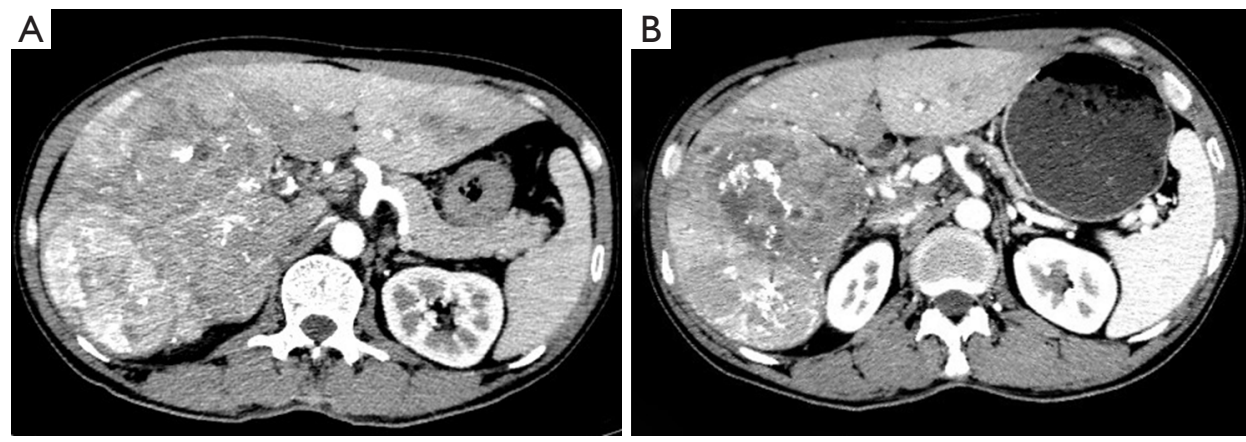

Figure 2 Representative images before and after apatinib treatment in case 1 . In this 48-year-old man with primary hepatocellular carcinoma, baseline contrast-enhanced CT (A) demonstrates multifocal tumors. After 8 weeks of therapy with apatinib. Multifocal tumors have decreased in density and size (B), showing greater homogeneity and better defined margins. Findings at follow-up CT represent response according to CT-morphologic criteria.
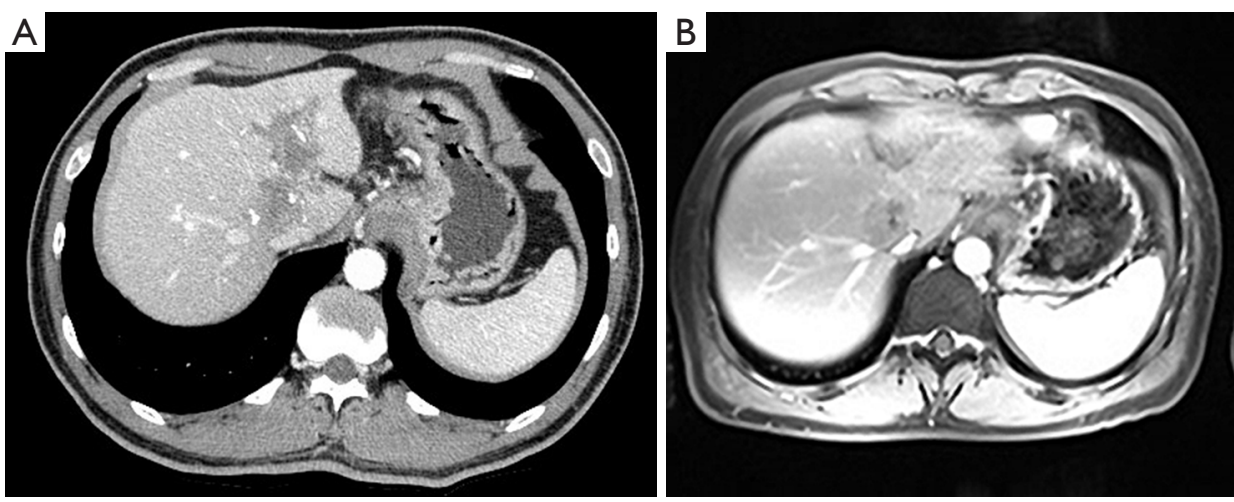

Figure 3 Representative images before and after apatinib treatment in case 2. In this 39-year-old man with primary hepatocellular carcinoma, baseline contrast-enhanced CT (A) demonstrates irregular nodular with portal vein tumor thrombus. After 8 weeks of therapy with apatinib, there is over $30 \%$ decrease in diameter of the sum of target lesions through arterial-enhancing CT and MRI.

The last date of follow-up was December 31, 2018. Median follow-up was 7.4 months (95\% CI, 5.7-14.7 months) in the apatinib group and 16.2 months (95\% CI, 13.1-20.7 months) in the sorafenib group.

Median PFS was 4.1 months (95\% CI, 3.2-7.4 months) for patients receiving apatinib and 3.6 months (95\% CI, 2.7-5.9 months) for patients receiving sorafenib. The 6-month and 1-year PFS rates were $26.4 \%$ and $13.2 \%$ for the apatinib group and $32.6 \%$ and $17.1 \%$ for the sorafenib group, respectively. Both groups had comparable PFS (HR 1.03 ; 95\% CI, 0.586-1.80; $\mathrm{P}=0.925$; Figure 4).

In total, $18(25 \%)$ of the 72 enrolled patients died [4 (15.4\%) of 26 in the apatinib group and 14 (30.4\%) of 46 in the sorafenib group]. The 6-month and 1-year OS rates were $95.8 \%$ and $62.0 \%$ for the apatinib group and $84.0 \%$ and $64.2 \%$ for the sorafenib group, respectively. The OS was not significantly different between the two groups [HR 1.15 (95\% CI 0.369-3.58); $\mathrm{P}=0.811$; Figure 4].

\section{Adverse events}

Treatment-emergent adverse events are listed in Table 3. The most common clinically relevant events were hand and foot syndrome [14 patients $(53.8 \%)$ in the apatinib group vs. 23 patients $(50 \%)$ in the sorafenib group], hypertension [13 patients $(50 \%)$ vs. 9 patients $(19.6 \%)]$, diarrhea [9 patients $(34.6 \%)$ vs. $13(28.3 \%)$ ], rash [6 patients $(23.1 \%)$ vs. 9 patients (19.6\%)], fatigue [6 patients $(23.1 \%) v s$. 10 patients $(21.7 \%)$ ], thrombocytopenia [6 patients $(23.1 \%)$ vs. 10 patients $(21.7 \%)$ ], proteinuria [5 patients $(19.2 \%)$ vs. 8 patients $(17.4 \%)$ ], and leukopenia [4 patients $(15.4 \%)$ vs. 7 patients $(15.2 \%)$ ]. Adverse events in both groups 

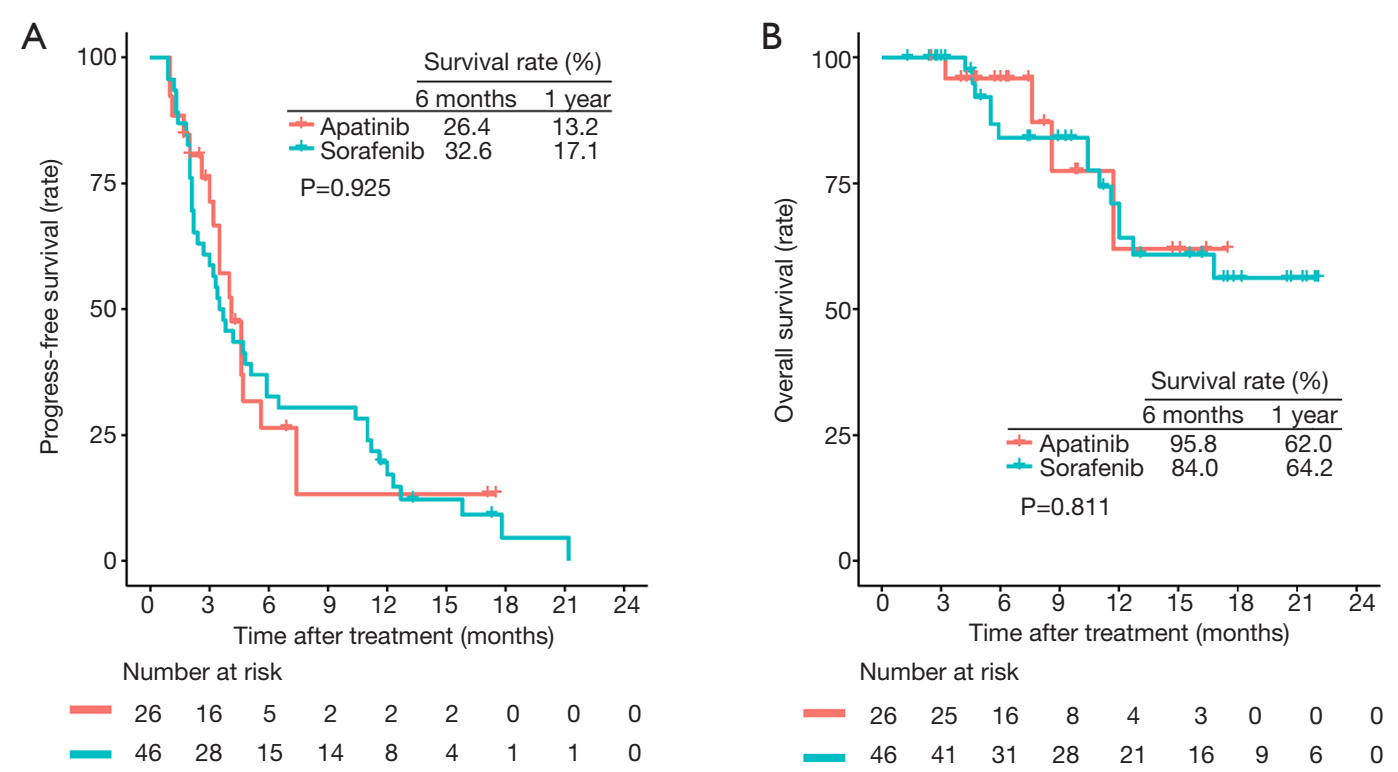

Figure 4 Kaplan-Meier estimated progression-free survival (A) and overall survival (B) curves of patients with hepatocellular carcinoma in the sorafenib and apatinib groups.

Table 3 Treatment-emergent adverse events by apatinib and sorafenib

\begin{tabular}{|c|c|c|c|c|}
\hline Adverse events & \multicolumn{2}{|c|}{ Apatinib group $(\mathrm{n}=26)$ [cases $(\%)]$} & \multicolumn{2}{|c|}{ Sorafenib group $(n=46)$ [cases $(\%)]$} \\
\hline Hand and foot syndrome & $14(53.8)$ & $3(11.5)$ & $23(50.0)$ & $3(6.5)$ \\
\hline Hypertension & $13(50.0)$ & $3(11.5)$ & 9 (19.6) & $3(6.5)$ \\
\hline Diarrhea & $9(34.6)$ & $2(7.7)$ & $13(28.3)$ & $2(4.3)$ \\
\hline Fatigue & $6(23.1)$ & 0 & $10(21.7)$ & 0 \\
\hline Elevated transaminase & $15(57.7)$ & 0 & $29(63.0)$ & 0 \\
\hline Proteinuria & $5(19.2)$ & $1(3.8)$ & $8(17.4)$ & $1(2.2)$ \\
\hline Vomiting & $3(11.5)$ & $1(3.8)$ & $6(13.0)$ & $1(2.2)$ \\
\hline Neutropenia & $3(11.5)$ & $1(3.8)$ & $6(13.0)$ & $1(2.2)$ \\
\hline Anemia & $7(26.9)$ & 0 & $13(28.3)$ & 0 \\
\hline
\end{tabular}

were predominantly grade 1 or 2 . Finally, 6 patients in the apatinib group and 8 patients in the sorafenib group who experienced grade 3 or 4 adverse events received dose adjustment. No treatment-related fatal adverse events were observed.

\section{Discussion}

This study compared the effectiveness of apatinib with sorafenib in patients with advanced HCC. Our study showed apatinib to be comparable to sorafenib in PFS 
and OS. We found that apatinib provided a statistically significant and clinically meaningful improvement in terms of ORR but not DCR, according mRECIST.

Currently, sorafenib is approved by the Food and Drug Administration of the United Status as the first-line therapy for advanced HCC. Unfortunately, sorafenib provides only a modest benefit, which necessitates alternative effective treatment regimens. Studies of patients treated with sorafenib yielded an ORR of only $2.0-3.3 \%(6,7,24)$. Our study also had a similar result in sorafenib group with $2 \%$ of patients (1/46) showing PR and half of the patients (23/46) having SD.

Apatinib was approved for the treatment of advanced gastric cancer in 2014 by the China Food and Drug Administration. Other studies have also reported that apatinib shows significant effectiveness in non-smallcell lung cancer and non-triple-negative metastasis breast cancer (25-27). In a phase II clinical trial of apatinib in patients with advanced HCC, the DCR was $48.57 \%$ and $37.25 \%$, while the time to progression (TTP) was 4.21 and 3.32 months in patients treated with a doses of 850 and $750 \mathrm{mg} /$ day, respectively (28). Apatinib-related adverse events were dose dependent. The DCR reached 57.7\% even with $500 \mathrm{mg}$ daily apatinib. A similar tumor response rate with an acceptable safety profile has been reported in advanced HCC patients treated with a low-dose of apatinib (21).

Current studies of apatinib in HCC mainly involve Asian populations $(20,21,28)$, in whom hepatitis $\mathrm{B}$ virus $(\mathrm{HBV})$ is still the largest attributable factor of HCC. In contrast, the majority of HCC cases in Europe and American are caused by the hepatitis $\mathrm{C}$ virus (HCV) and alcohol. Meta-analysis data suggest that HCC patients with HCV infection may have a better response to sorafenib than those with other underlying causes of cirrhosis (29). However, the difference in tumor response to apatinib among Asian and Western population remains unknown.

This study was primarily designed to compare PFS evaluated by mRECIST after treatment of apatinib and sorafenib. The mRECIST has been proven to be more robust than the RECIST in assessing the response of tumor in HCC patients treated with molecularly targeted therapy, as devascularization is a better hallmark of response than shrinkage of tumor in HCC (23). In our study, the ORR was significantly higher in the apatinib group than in the sorafenib group.Apatinib is a highly selective VEGFR-2 inhibitor and thus may exert a stronger effect on antiangiogenesis. Short-term treatment of apatinib could cause tumors to shrink dramatically by blocking the blood supply. In addition, apatinib may rescue more liver surgeries or ablations in patients with initially unresectable HCC with a higher ORR. Our results strongly support a prospective and randomized study to explore the value of apatinib in conversion therapy.

However, given the retrospective nature of the, current study, some limitations should be kept in mind. First, this study only included a relatively small sample size of patients treated with apatinib. There were only 18 death events, so risk factors associated with long-term survival could not be assessed. In addition, retrospective study does not always guarantee a complete data set, and thus missing data might have hindered some aspects of the analysis.

\section{Conclusions}

Apatinib exhibited superior objective response by mRECIST and comparable PFS and OS compared with sorafenib in advanced HCC. Further prospective randomized studies are required to clarify the benefit of apatinib.

\section{Acknowledgments}

Funding: This work was supported by grants from the Science and Technology Program of Guangzhou, China (No. 201803010071), Science and Technology Planning Project of Guangdong Province, China [Grant Number 2015B010125003], also by Science and Technology Program of Guangzhou, China [Grant Number 201704020133].

\section{Footnote}

Reporting Checklist: The authors have completed the STROBE reporting checklist. Available at http://dx.doi. org/10.21037/atm-20-5298

Data Sharing Statement: Available at http://dx.doi. org/10.21037/atm-20-5298

Conflicts of Interest: All authors have completed the ICMJE uniform disclosure form (available at http://dx.doi. org/10.21037/atm-20-5298). The authors have no conflicts of interest to declare.

Ethical Statement: The authors are accountable for all aspects of the work in ensuring that questions related 


\section{Page 8 of 9}

to the accuracy or integrity of any part of the work are appropriately investigated and resolved. The study protocol conformed to the ethical guidelines of the Declaration of Helsinki (as revised in 2013), and the Ethics Committee of Sun Yat-Sen University Cancer Center approved this study (No. YB2018-08). Because of the retrospective nature of the research, the requirement for informed consent was waived.

Open Access Statement: This is an Open Access article distributed in accordance with the Creative Commons Attribution-NonCommercial-NoDerivs 4.0 International License (CC BY-NC-ND 4.0), which permits the noncommercial replication and distribution of the article with the strict proviso that no changes or edits are made and the original work is properly cited (including links to both the formal publication through the relevant DOI and the license). See: https://creativecommons.org/licenses/by-nc-nd/4.0/.

\section{References}

1. Villanueva A. Hepatocellular Carcinoma. N Engl J Med 2019;380:1450-62.

2. Bray F, Ferlay J, Soerjomataram I, et al. Global cancer statistics 2018: GLOBOCAN estimates of incidence and mortality worldwide for 36 cancers in 185 countries. CA Cancer J Clin 2018;68:394-424.

3. Boland P, Wu J. Systemic therapy for hepatocellular carcinoma: beyond sorafenib. Chin Clin Oncol 2018;7:50.

4. Kotewall CN, Cheung TT. Optimizing hepatectomy for hepatocellular carcinoma in Asia-patient selection and special considerations. Transl Gastroenterol Hepatol 2018;3:75.

5. Miura H, Miyazaki T, Kuroda M, et al. Increased expression of vascular endothelial growth factor in human hepatocellular carcinoma. J Hepatol 1997;27:854-61.

6. Llovet JM, Ricci S, Mazzaferro V, et al. Sorafenib in advanced hepatocellular carcinoma. N Engl J Med 2008;359:378-90.

7. Cheng AL, Kang YK, Chen Z, et al. Efficacy and safety of sorafenib in patients in the Asia-Pacific region with advanced hepatocellular carcinoma: a phase III randomised, double-blind, placebo-controlled trial. Lancet Oncol 2009;10:25-34.

8. Cheng AL, Kang YK, Lin DY, et al. Sunitinib versus sorafenib in advanced hepatocellular cancer: results of a randomized phase III trial. J Clin Oncol 2013;31:4067-75.

9. Johnson PJ, Qin S, Park JW, et al. Brivanib versus sorafenib as first-line therapy in patients with unresectable,
He et al. Apatinib versus sorafenib in hepatocellular carcinoma

advanced hepatocellular carcinoma: results from the randomized phase III BRISK-FL study. J Clin Oncol 2013;31:3517-24.

10. Cainap C, Qin S, Huang WT, et al. Linifanib versus Sorafenib in patients with advanced hepatocellular carcinoma: results of a randomized phase III trial. J Clin Oncol 2015;33:172-9.

11. Zhu AX, Rosmorduc O, Evans TR, et al. SEARCH: a phase III, randomized, double-blind, placebo-controlled trial of sorafenib plus erlotinib in patients with advanced hepatocellular carcinoma. J Clin Oncol 2015;33:559-66.

12. Zhu AX, Kang YK, Yen CJ, et al. Ramucirumab after sorafenib in patients with advanced hepatocellular carcinoma and increased $\alpha$-fetoprotein concentrations (REACH-2): a randomised, double-blind, placebocontrolled, phase 3 trial. Lancet Oncol 2019;20:282-96.

13. Abou-Alfa GK, Meyer T, Cheng AL, et al. Cabozantinib in Patients with Advanced and Progressing Hepatocellular Carcinoma. N Engl J Med 2018;379:54-63.

14. Kudo M, Finn RS, Qin S, et al. Lenvatinib versus sorafenib in first-line treatment of patients with unresectable hepatocellular carcinoma: a randomised phase 3 noninferiority trial. Lancet 2018;391:1163-73.

15. Bruix J, Qin S, Merle P, et al. Regorafenib for patients with hepatocellular carcinoma who progressed on sorafenib treatment (RESORCE): a randomised, double-blind, placebo-controlled, phase 3 trial. Lancet 2017;389:56-66.

16. Fontanella C, Ongaro E, Bolzonello S, et al. Clinical advances in the development of novel VEGFR2 inhibitors. Ann Transl Med 2014;2:123.

17. Chen W, Yu J, Zhang Y, et al. First-line application of apatinib combined with S-1 based on peripheral circulating tumor cell screening to treat advanced gastric adenocarcinoma: a case report. Ann Transl Med 2019;7:181.

18. Tian S, Quan H, Xie C, et al. YN968D1 is a novel and selective inhibitor of vascular endothelial growth factor receptor-2 tyrosine kinase with potent activity in vitro and in vivo. Cancer Sci 2011;102:1374-80.

19. Li J, Qin S, Xu J, et al. Apatinib for chemotherapyrefractory advanced metastatic gastric cancer: results from a randomized, placebo-controlled, parallel-arm, phase II trial. J Clin Oncol 2013;31:3219-25.

20. Yu WC, Zhang KZ, Chen SG, et al. Efficacy and Safety of apatinib in patients with intermediate/advanced hepatocellular carcinoma: A prospective observation study. Medicine (Baltimore) 2018;97:e9704.

21. Kong Y, Sun L, Hou Z, et al. Apatinib is effective 
for treatment of advanced hepatocellular carcinoma. Oncotarget 2017;8:105596-605.

22. Edeline J, Boucher E, Rolland Y, et al. Comparison of tumor response by Response Evaluation Criteria in Solid Tumors (RECIST) and modified RECIST in patients treated with sorafenib for hepatocellular carcinoma. Cancer 2012;118:147-56.

23. Lencioni R, Llovet JM. Modified RECIST (mRECIST) assessment for hepatocellular carcinoma. Semin Liver Dis 2010;30:52-60.

24. Abou-Alfa GK, Schwartz L, Ricci S, et al. Phase II study of sorafenib in patients with advanced hepatocellular carcinoma. J Clin Oncol 2006;24:4293-300.

25. Song Z, Yu X, Lou G, et al. Salvage treatment with apatinib for advanced non-small-cell lung cancer. Onco Targets Ther 2017;10:1821-5.

Cite this article as: $\mathrm{He} W$, Liao L, Hu D, Li B, Wang C, Qiu J, Liao Y, Liu W, Yang Z, Zhang Y, Yuan Y, Li K, Yuan Y, Zheng Y. Apatinib versus sorafenib in patients with advanced hepatocellular carcinoma: a preliminary study. Ann Transl Med 2020;8(16):1000. doi: 10.21037/atm-20-5298
26. $\mathrm{Hu} \mathrm{X}, \mathrm{Cao} \mathrm{J}, \mathrm{Hu} \mathrm{W}$, et al. Multicenter phase II study of apatinib in non-triple-negative metastatic breast cancer. BMC Cancer 2014;14:820.

27. Ding L, Li QJ, You KY, et al. The Use of Apatinib in Treating Nonsmall-Cell Lung Cancer: Case Report and Review of Literature. Medicine (Baltimore) 2016;95:e3598.

28. Qin S. Apatinib in Chinese patients with advanced hepatocellular carcinoma: A phase II randomized, openlabel trial. J Clin Oncol 2014;32:abstr 4019.

29. Jackson R, Psarelli EE, Berhane S, et al. Impact of Viral Status on Survival in Patients Receiving Sorafenib for Advanced Hepatocellular Cancer: A Meta-Analysis of Randomized Phase III Trials. J Clin Oncol 2017;35:622-8.

(English Language Editor: J. Gray) 